\title{
IL SENATO DI PIEMONTE E IL CASO REVELLO (1723). UN CONFLITTO ISTITUZIONALE AL VERTICE DELLO STATO SABAUDO NEL CONTESTO DEL RIFORMISMO DI VITTORIO AMEDEO II
}

\author{
SENATE OF PIEDMONT AND REVELLO CASE (1723). \\ AN INSTITUTIONAL CONFLICT AT THE TOP OF KINGDOM \\ OF SARDINIA IN THE CONTEXT OF VICTOR AMADEUS II'S REFORMS
}

\author{
Matteo Traverso \\ Università degli Studi di Torino
}

\begin{abstract}
English: Victor Amadeus II is generally recognized by historiography as the initiator of a major administrative and judicial reorganization, begun after the Peace of Utrecht and the conclusion of the War of the Spanish Succession. This has been a long and complex process, which also led to the issuing of a new body of laws: the Leggi e Costituzioni di Sua Maestà il Re di Sardegna of 1723. Victor Amadeus II's action also involved the Savoy judicial system. Recent studies have analysed the reforms carried out by this sovereign during his long reign on the most important existing magistracies in the Kingdom of Sardinia (the Senates and the Camera dei Conti). This article aims to examine one of the most critical episodes in the institutional and legal history of Savoy, namely the conflict between 1722 and 1723 between Victor Amadeus II and the Senate of Piedmont. This clash led to the replacement of a significant number of Turin senators including President Paolo Francesco Leone. The king came into conflict with his Senate due to the acquittal that this magistrate gave to Carlo Lorenzo Revello, a country guard who was caught armed near Mondovì. In fact, an edict of 1699 punished the conduct of carrying weapons in those places with the death penalty. The Senate, however, ignored the king's rule and did not condemn Revello: according to the judge's reasoning, Revello exercised a profession that made it necessary to carry arms. Thus, a close correspondence between the Senate of Turin and the king began: on the one hand, the sovereign insisted on the application of his edict of 1699, on the other, the judiciary of Turin upheld Revello's innocence and good faith.
\end{abstract}

The issues that were at the basis of this institutional dispute were two. One of a legal nature and one of a more political nature. The legal one contrasted two opposing views of law: on the one hand the Senate, in favour of the use of equity in the application of law, and on the other the king interested in imposing his will without further discussion. In practice, we have a more formalistic and stringent vision of the law that seeks to impose itself on the Savoy judiciary, used to exercising a certain arbitrariness in the interpretation of laws.

However, this clash at the top of the Savoy state was not caused only by legal reasons.

* Italian Review of Legal History, 7 (2021), n. 4, pagg. 109-130

* https://riviste.unimi.it/index.php/irlh/index

* ISSN 2464-8914 - DOI 10.54103/2464-8914/16886. Articolo pubblicato sotto Licenza CC-BY. 
This strong contrast also concealed a political question: Victor Amadeus II was a reforming sovereign, but with absolutist and centralizing tendencies; it was certainly among his objectives to reaffirm his position of strength against a Senate that claimed to have a lot of freedom.

A fairly minor legal question became a very sensitive political issue. Several members of the Senate were in fact convinced of the specious nature of the affair and of the accusations made by the king. For example, Maurizio Ignazio Graneri, one of the senators directly affected by the Victor Amadeus II's sanctions, argued that the Revello case was just the king's excuse to dissolve the Senate and fill it with new, more loyal members.

The article begins with an analysis of this conflict at the head of the State and then extends the study to the complex relationship between political and judiciary power during the reign of Victor Amadeus II. The sovereign grievances, the defences of the senators and the opinions of other jurists consulted by the king allow us to reconstruct this clash but also to investigate a more important question: how far could the interpretative power of a sovereign court on royal legislation go?

Keywords: Victor Amadeus II; Senate of Turin; Judicial interpretation; Revello case; Kingdom of Sardinia.

Abstract Italiano: L'articolo intende approfondire la contrapposizione che si ebbe a cavallo tra il 1722 ed il 1723 tra Vittorio Amedeo II e il Senato di Piemonte a causa dell'assoluzione che questa magistratura diede ad una guardia campestre che era stata sorpresa armata nel mandamento di Mondovì in violazione di un editto regio. Le rimostranze sovrane, le difese dei senatori e i pareri degli altri giuristi interpellati dal re consentono di ricostruire questo scontro ma consentono anche di indagare una questione più importante: fino a che punto poteva arrivare il potere interpretativo di una corte sovrana sulla normativa regia?

Parole chiave: Vittorio Amedeo II; Senato di Piemonte; Interpretazione giudiziaria; Caso Revello; Regno di Sardegna.

Sommario: 1. Introduzione. - 2. Lo scontro tra Vittorio Amedeo II ed il Senato di Piemonte: un problema di interpretazione. - 3. Le conseguenze del caso Revello nel contesto della riforma delle magistrature sabaude. - 4. Conclusioni. Vittorio Amedeo II e il caso Revello: despota o innovatore? La lettura della storiografia sabauda ottocentesca.

\section{Introduzione}

Negli ultimi vent'anni del XVII secolo (1680-1699) il ducato di Savoia venne interessato da una serie di insorgenze armate che si susseguirono nel territorio del Monregalese. Questi eventi sono passati alla storia come Guerra del Sale ${ }^{1}$ in quanto le prime rivolte ebbero origine a seguito della scelta di applicare anche

${ }^{1}$ Da un punto di vista militare, questa guerra ha avuto due episodi cruciali: il 1681 con la battaglia di Montaldo, che porterà ad una pacificazione con Torino nel 1682, e il 1698- 
a Mondovì la gabella su questo prodotto, disattendendo i secolari privilegi di cui quella comunità aveva goduto².

Nel 1699, alla fine di questo lungo periodo di instabilità politica e sociale, il giovane duca Vittorio Amedeo II emanò un editto particolarmente severo per «frenare ed impedire ogni ulteriore sommossa nel mandamento di Mondovi»». Questa normativa prevedeva una serie di prescrizioni particolarmente stringenti tra cui il divieto di ricostruire gran parte degli edifici e delle strutture che erano stati rasi al suolo nel recente conflitto, il divieto generalizzato di portare armi o anche solo detenerle in casa senza aver ottenuto una specifica autorizzazione e ancora diverse limitazioni (più incisive del solito, anche per i tempi) alla libertà di associazione ${ }^{4}$.

Pacificato il Monregalese, nel primo decennio del XVIII secolo il ducato venne immediatamente interessato da un nuovo conflitto (questa volta esterno), finendo coinvolto nella Guerra di Successione Spagnola. II trattato di Utrecht (1713) inaugurò finalmente un periodo relativamente lungo di pace ${ }^{5}$, chiudendo idealmente quasi un secolo di conflitti e consentendo a Vittorio Amedeo II di dare avvio ad una significativa operazione di riorganizzazione amministrativa e giudiziaria dello Stato.

Pur rigettando la rappresentazione eccessivamente agiografica della figura del primo re di Sardegna presentata dalla storiografia meno recente, è indubbio che il nuovo sovrano, assumendo il governo nel 1684 dalla reggente Maria Giovan-

1699 che porterà alla definitiva e totale affermazione del potere ducale in quelle zone. Facendo riferimento a questi due momenti si è perciò a lungo parlato nella storiografia di una "prima" e di una "seconda" Guerra del Sale. In realtà studi più recenti ed approfonditi hanno messo in evidenza che questo ventennio della storia sabauda è stato continuamente caratterizzato da rivolte e insurrezioni (seppur minori) che ne rendono necessaria un'analisi ed una considerazione complessiva ed unitaria; Lombardi, 1962, pp. 22-23 (in nota 47). Per una bibliografia essenziale di questa guerra si rimanda a Lombardi, 2003, pp. 185-206, ed ai contributi pubblicati nei tre volumi curati da Giorgio Lombardi ed editi nel 1986 (La Guerra del Sale (1680-1699). Rivolte e frontiere del Piemonte barocco, I-II-III, Milano, Franco Angeli).

2 Sui privilegi del comune di Mondovì, a cominciare dal momento in cui è entrato nella sfera di influenza dei Savoia in epoca medievale (1347), e sulla crescente pretesa ducale di estendere anche in quei luoghi una propria gabella del sale si veda Pene Vidari, 1986, pp. 367-385.

3 Ordine di S.A.R., col quale si danno varii provvedimenti per frenare ed impedire ogni ulteriore sommossa nel mandamento di Mondovì, pubblicato in Duboin, 1830, pp. 26-34.

4 Ivi, pp. 28-34. Per una breve analisi di questo editto si veda Lange, 1986, pp. 71-75.

${ }_{5}$ Sulle conseguenze di questa pace, grazie alla quale i Savoia ottennero il titolo regio oltre che un notevole ampliamento territoriale, si veda Bianchi, Merlotti, 2017, pp. 42-43 e Ricuperati, 2016, pp. 17-24. Per una analisi interdisciplinare di questo evento si vedano i contributi pubblicati nel volume edito nel 2014 e curato da Gustavo Mola di Nomaglio e Giancarlo Melano, Utrecht 1713. I trattati che aprirono le porte d'Italia ai Savoia. Studi per il terzo centenario, Centro Studi Piemontesi, Torino. 
na Battista di Savoia Nemours, sia riuscito a portare a compimento molte delle riforme politiche e amministrative pensate dai suoi predecessori ${ }^{6}$. Oltre alla riorganizzazione della legislazione patria, realizzata nel 1723 con l'emanazione delle Regie Costituzioni (oggetto poi di altre due edizioni del 1729 e del 1770$)^{7}$, tra gli obiettivi del re rientrava anche la razionalizzazione e l'accentramento dell'ordinamento giudiziario. Quest'ultimo intervento non era tuttavia facile da realizzare in quanto presupponeva un efficientamento delle supreme magistrature dello Stato (con una miglior definizione delle rispettive sfere di competenza e delle loro attribuzioni) e, conseguentemente, lo scontro con l'élite subalpina che dirigeva quelle stesse istituzioni e che era assai gelosa delle proprie secolari prerogative.

In questo contesto, uno dei momenti più delicati nel rapporto tra il sovrano ed uno dei principali organi giudiziari sabaudi, ovvero il Senato di Torino ${ }^{8}$, si ebbe nel 1723 e venne originato da un caso giudiziario in cui si avvertiva nitidamente l'eco della passata Guerra del Sale.

Si trattava (mutuando la felice espressione utilizzata da Enrico Genta) del cd. "affare Revello" istituzionale tanto per l'oggetto (che certo non interessava questioni di Stato) quanto per il livello sociale dei soggetti coinvolti.

La vicenda ebbe inizio il 13 dicembre 1721, quando Carlo Lorenzo Revello, fiscale di Monastero di Vasco (in provincia di Mondovì) venne arrestato nelle vicinanze di una cappella dedicata a Santa Lucia, sita nei pressi dell'attuale comune di Villanova Mondovì. Essendo stato trovato armato di fucile e pistola egli venne accusato di aver violato l'art. 4 del già citato editto del 4 luglio $1699^{10}$ che proibiva in tutto il mandamento di Mondovì, "sotto pena della vita» ${ }^{11}$, di portare armi da fuoco.

Il giorno successivo all'arresto del Revello (14 dicembre) il podestà di Villanova, Franco Lanza, ne diede immediatamente informazione al Senato di Piemonte che era la magistratura territorialmente competente a conoscere i reati commessi in quel mandamento ${ }^{12}$. La suprema corte piemontese rispose il 22 dicembre chiedendo alcuni ragguagli sulla vicenda, richiesta rinnovata il 20 gennaio 1722 al

\footnotetext{
${ }^{6}$ Ricuperati, 2002, p. XXVIII.

7 Sul processo di formazione delle Leggi e Costituzioni di Sua Maestà si veda Viora, 1928, passim e Micolo, 1984, passim.

8 Sulla storia del Senato di Piemonte, oltre ai contributi contenuti nei volumi editi nel 2001 da Gian Savino Pene Vidari (Les Sénats de la Maison de Savoie. Ancien régime - Restauration, Giappichelli, Torino) e nel 2016 da Françoise Briegel e Sylvain Milbach (Les Sénats des Etats de Savoie. Circulations des pratiques judiciaires, des magistrats, des normes (XVI-XIX siècles), Carocci, Roma), si veda in particolare: Dionisotti, 1881, pp. 67-80, 125-146; Genta, 1983, pp. 1-131; Mongiano, 1991, pp. 161-191; Casana, 1995, pp. 1-54.

9 Genta, 1986, p. 18.

10 Duboin, 1830, pp. 26-34.

11 Ivi, p. 30.

12 Genta, 1986, p. 392.
} 
nuovo podestà Antonio Mamino ${ }^{13}$.

Ma chi era l'arrestato? Sommarie informazioni si possono a questo proposito trarre dagli atti delle indagini che l'Ufficio del fisco di Mondovì condusse nei mesi successivi alla sua cattura. Nel corso degli interrogatori a cui fu sottoposto, Revello dichiarò innanzi tutto di avere trentotto anni, di non saper scrivere, di non aver beni salvo la modesta dote della moglie e di svolgere da circa sette anni il ruolo di "fiscale" nel luogo di Monastero di Vasco ${ }^{14}$.

Dalla lettura di questi atti emerge nel complesso la figura di una persona di modestissima estrazione sociale e piuttosto ingenua, ma nondimeno apprezzata dalla comunità in cui prestava servizio: una "persona di buona voce, e fama, e timorata dell'humana, e Divina giustizia [...] benché zelante della giustizia, idiota però, povera, e rurale, e carica di figliuoli» ${ }^{15}$.

Come si cercherà di evidenziare nelle pagine seguenti, da questo fatto all'apparenza secondario si aprì una delle più gravi crisi istituzionali del regno di Vittorio Amedeo II che portò ad esiti drammatici per i magistrati che ne vennero coinvolti.

\section{Lo scontro tra Vittorio Amedeo II ed il Senato di Piemonte: un problema di interpretazione}

L'imputazione contestata a Revello era grave e, se confermata in giudizio, avrebbe comportato necessariamente l'applicazione della pena capitale.

Essendo stato arrestato in piena flagranza di reato, la violazione dell'editto del 1699 non poteva di per sé essere contestata ed infatti la sua difesa si fondava su due diversi punti: egli da un lato sostenne di non aver compreso (incolpevolmente) l'esatto tenore dell'editto e dall'altro affermò di girare armato per salvaguardare la propria incolumità dai rischi derivanti dalla propria professione ${ }^{16}$.

Quanto al primo aspetto, in sede di interrogatorio il fiscale di Monastero di Vasco dichiarò di non essere a conoscenza del fatto che anche chi svolgeva (come lui) funzioni latu sensu pubbliche avrebbe comunque dovuto ottenere una dispensa speciale per poter portare legittimamente armi nella provincia di Mondovì.

In effetti in tutte le altre province sabaude i fiscali come Revello erano autorizzati a girare armati semplicemente in ragione delle loro nomine. Tuttavia la provincia di Mondovì faceva eccezione proprio a causa dei recenti tumulti che avevano indotto Vittorio Amedeo II ad emanare ed applicare sulla questione una

\footnotetext{
${ }^{13}$ Archivio di Stato di Torino (ASTo), Sez. Corte, Materie Giuridiche, Materie giuridiche per categorie, Senato di Piemonte, m. 2, n. 33, 1, Relazione della causa criminale, e fatti concernenti la medesima contro Carlo Lorenzo Revello preteso fiscale del luogo di Monastero di Vasco Provincia del Mondovì, e detenuto per porto d'armi, f. 2 v.

${ }^{14}$ Ivi, f. 3 v.

15 Ivi, f. 4 v.

${ }^{16}$ ASTo, Sez. Corte, Materie Giuridiche, Materie giuridiche per categorie, Senato di Piemonte, m. 2, n. 33, 1, Relazione della causa criminale, e fatti concernenti la medesima, cit., f. 4 r.
} 
legislazione assai più stringente ${ }^{17}$.

Ad una prima analisi potrebbe apparire strano che l'arrestato non fosse consapevole della specifica disciplina vigente nel luogo in cui aveva sempre vissuto ed operato. Bisogna tuttavia considerare due aspetti: da un lato la bassa estrazione sociale e culturale del Revello e dall'altro il carattere assai più "fattuale" che giuridico della sua professione. Nel corso delle indagini infatti il fisco di Mondovì fece notare che la stessa nomina di Revello presentava diverse irregolarità: le uniche patenti prodotte in giudizio risalivano all'anno precedente (ovvero al 1721 ed erano sottoscritte dal conte Giuseppe Torrini, Vassallo di Monastero) ed oltretutto non erano state mai approvate dal presidente della Provincia ${ }^{18}$. In relazione agli anni di servizio precedenti non vennero mai prodotte patenti di nomina valide, nonostante tutti gli ufficiali di giustizia del luogo fossero ben a conoscenza di quale fosse la sua professione ${ }^{19}$. Avendolo sempre fatto senza alcuna contestazione, è quindi effettivamente possibile che Revello ritenesse in buona fede di poter legittimamente girare armato in qualità di fiscale anche perché, su questo punto, l'editto del 1699 risultava essere, ormai da diversi anni, largamente disatteso ${ }^{20}$.

La seconda argomentazione difensiva svolta da Revello faceva invece perno sulla circostanza che non fosse in alcun modo possibile svolgere la sua professione senza portare armi. Era semplicemente inconcepibile che il contrasto alla criminalità campestre potesse essere svolto da guardie disarmate. I rischi erano molteplici, tanto che l'accusato affermò di essersi recato armato alla cappella di Santa Lucia (in una zona tra l'altro non compresa nella sua "giurisdizione" di fiscale) proprio per proteggersi da alcuni banditi del luogo, dai quali aveva recentemente ricevuto delle minacce di morte. Nella relazione degli atti della causa di Revello su questa circostanza viene specificato quanto segue:

non ha avuto cuore di andarci disarmato, perché Giuseppe Reynoso, et un altro, di cui dice non saperne il nome, ne cognome, ambi però delle fini di Frabosa, e banditi, gl'avevano il giorno seguente alla festa di S. Morizio all'hora scorsa, e per mezzo di Carlo Vincenzo Roatta, fatto sapere che ovunque l'avessero ritrovato, lo volevano uccidere ${ }^{21}$.

Per via del suo peculiare lavoro i timori del Revello potevano dirsi giustificati. La testimonianza resa dal podestà di Monastero di Vasco confermò che grazie al suo intervento erano stati catturati nel corso del tempo diversi banditi che infe-

17 Genta, 1986, pp. 388-389.

${ }_{18}$ ASTo, Sez. Corte, Materie Giuridiche, Materie giuridiche per categorie, Senato di Piemonte, m. 2, n. 33, 1, Relazione della causa criminale, e fatti concernenti la medesima, cit., f. 5 v.

19 Ibidem.

20 Lange, 1986, pp. 72-73.

${ }^{21}$ ASTo, Sez. Corte, Materie Giuridiche, Materie giuridiche per categorie, Senato di Piemonte, m. 2, n. 33, 1, Relazione della causa criminale, e fatti concernenti la medesima, cit., f. 4 v. 
stavano quelle zone e che, conseguentemente, il rischio di vendette e ritorsioni era concreto.

Delle due ragioni addotte a sua difesa da Revello quella che inizialmente colpì maggiormente il Senato di Torino fu certamente la seconda. In effetti una piena applicazione dell'editto del 1699, anche nei confronti degli ufficiali di giustizia della provincia di Mondovì, avrebbe nei fatti impedito a questi ultimi di poter contrastare le insorgenze criminali presenti in loco.

Questa argomentazione convinse pienamente la corte torinese; tuttavia i senatori erano ben consapevoli che disattendere de plano il testo dell'editto regio (che con chiarezza non esentava nessuna categoria dalla sua applicazione) avrebbe rappresentato uno strappo istituzionale irreparabile. Proprio per tale ragione il 19 dicembre 1722, appena ricevuta la causa, la suprema magistratura sabauda tentò in prima battuta di evitare uno scontro frontale dall'esito imponderabile e sottopose la questione al re, dando segno, con le parole che di seguito si riportano, di cercare una mediazione sull'interpretazione della citata normativa:

Sovra ciò attesa la dubbietà dell'articolo, e necessità che hanno simili officiali di giustizia di portare le armi abbiamo creduto d'aver giusto motivo d'esplorare dalla somma clemenza di Vostra Reale Maestà se sia stata mente sua, che detti officiali di giustizia a differenza di quel che si osserva nel rimanente dei suoi Stati siano compresi nella proibizione portata da detto editto $[. . .]^{22}$.

La speranza era di riuscire ad indurre Vittorio Amedeo II ad "allargare le maglie" di un editto risalente ad oltre vent'anni prima, emanato in un contesto storico e sociale ben diverso (e assai più turbolento) da quello del caso Revello.

Tuttavia il 13 gennaio 1723 il sovrano confermò la piena vigenza di quel divieto per tutti gli abitanti della provincia di Mondovì (compresi i fiscali come l'arrestato), inviando al Senato una risposta di tale tenore:

Tutto che gradiamo che per l'intelligenza dei nostri editti abbiate avuto ricorso a noi, già però vi deve essere nota la nostra intenzione circa il divieto delle armi per il mandamento del Mondovì, mentre non solo nell'editto delli 21 febbraio 1709 contenente la proibizione di detenzione d'armi per il generale dei nostri Stati preservassimo le precedenti nostre disposizioni per quella Provincia, ma altresì perché si è colà d'ordine nostro fatto ripubblicare da quel Governatore l'editto delli 4 luglio 1699, gionta pure la lettera da noi scrittaci su tal particolare sin dalli 15 dell'or caduto novembre, onde esso editto delli 4 luglio non eccettuando alcuno, e quello delli 21 febbraio eccettuandone molti, niente osta, perché rispetto ad una Provincia non dobbiate osservare l'editto particolare, ed il generale per il rimanen-

22 ASTo, Sez. Corte, Materie Giuridiche, Materie giuridiche per categorie, Senato di Piemonte, m. 2, n. 33, 14, Lettera del Senato di Piemonte a S.M. con cui espone la sua dubbietà se sia mente della MS che gl'ufficiali di giustizia della Provincia del Mondovì siano compresi nella proibizione del porto d'armi dell'Editto 1699, e ciò a riguardi del fiscale Revello detenuto, 19 dicembre 1722. 
te dei nostri Stati sottoposto alla vostra giurisdizione ${ }^{23}$.

Come si evince da questa risposta il re di Sardegna evitò di entrare nel merito della questione sollevata dal Senato circa l'opportunità e la ragionevolezza di una norma che vietava agli ufficiali di giustizia di detenere e portare armi, ma si limitò a ribadire il particolare regime giuridico che vigeva nel Monregalese, concludendo la sua risposta con un laconico «Tanto dunque eseguite ${ }^{24}$.

Nonostante la chiara manifestazione della volontà regia, il Senato di Torino decise comunque di assolvere all'unanimità Revello dall'accusa di aver violato l'editto del 1699. Tuttavia, probabilmente nell'estremo tentativo di lasciare ancora margine per una ricomposizione con la corona ${ }^{25}$, i giudici decisero di non procedere alla pubblicazione della sentenza prima di aver esposto al sovrano le motivazioni della loro scelta e di averne atteso la risposta.

Le ragioni giuridiche che spinsero i giudici sabaudi ad assolvere l'accusato vennero quindi analiticamente esposte a Vittorio Amedeo II in una lettera del 19 aprile $1723^{26}$. A differenza di quanto fatto nella prima rappresentanza del 19 dicembre non si poteva però più mettere in dubbio che il divieto di porto d'armi si dovesse applicare anche agli ufficiali di giustizia. Dopo la prima risposta del re questo era infatti ormai pacifico. Il Senato tuttavia riconobbe nella condotta del Revello «una scusabile credulità» e la presenza «di motivi tali, che l'hanno potuto porre in istato di buona fede, e così al di fuori di quel dolo, che si richiede per farlo incorrer nella pena» ${ }^{27}$.

${ }^{23}$ ASTo, Sez. Corte, Materie Giuridiche, Materie giuridiche per categorie, Senato di Piemonte, m. 2, n. 33, 1, 15, Copia di biglietto di R.S.M., al Senato, con cui gli risponde che non eccettuando alcuno la disposizione dell'Editto 1699 quale deve osservarsi nella Provincia del Mondovì non deve rimanergli dubbio alcuno a riguardo del fiscal Revello, 13 gennaio 1723. L'editto "generale" sul porto delle armi del 21 febbraio 1709, a cui Vittorio Amedeo II si riferiva in questo biglietto, faceva effettivamente salva la speciale disciplina vigente a Mondovì: «Non vogliamo però, che per la disposizione del presente, benché universale, s'intendano innovate in alcuna parte le precedenti nostre disposizioni, pohibitioni, et ordini per la città, terre, e provincia di Mondovì, e suo mandamento, rispetto a' quali luoghi, sudditi, et habitanti in essi, si osserverà tanto circa le prohibitioni, che pene, il portato dalle nostre precedenti provvisioni, et ordini»; Duboin, 1830, Editto di S.A.R. sulla proibizione del porto elle armi, e loro fabbricazione e vendita, sotto le eccettuazioni in esso contenute, pp. 205-209 (citazione a p. 208).

${ }^{24}$ ASTo, Sez. Corte, Materie Giuridiche, Materie giuridiche per categorie, Senato di Piemonte, m. 2, n. 33, 1, 15, Copia di biglietto di R.S.M., cit.

${ }^{25}$ Genta, 1986, p. 392.

${ }^{26}$ ASTo, Sez. Corte, Materie Giuridiche, Materie giuridiche per categorie, Senato di Piemonte, m. 2, n. 33, 16, Lettera del Senato di Piemonte a S.M. con cui le rappresenta che non ostante la precedente sua regia dichiarazione ha per i motivi che allega assolto il fiscale Revello con voti uniformi dalla pena dell'Editto 1699, sospendendo però di mettere la sentenza in scritti sino a nuovo ordine della M.S., 19 aprile 1723.

27 Ibidem. 
Per rafforzare la tesi della mancanza di dolo nella violazione del Revello, la magistratura sabauda indicò una serie di elementi che ricavò interpretando teleologicamente l'editto del 1699. In particolare: se il fine di questa legge era garantire il mantenimento della quiete e della tranquillità nella provincia di Mondovì, come indicato nel proemio della stessa ${ }^{28}$, era fisiologico che gli ufficiali di giustizia (sui quali per primi ricadeva in concreto questo compito) potessero comprensibilmente ritenere (seppur erroneamente) di non incorrere nei divieti relativi alle armi in quanto, come scrissero i senatori al sovrano, «quando non fosse permesso a simili servienti di giustizia il porto delle armi, vi sarebbe minor sicurezza di quel che vi sia nelle altre Provincie ${ }^{29}$. Ad avviso del Senato questa conclusione era inoltre suffragata dal diritto delle genti (per il quale gli ufficiali di giustizia hanno sempre il diritto di essere armati) e dalle consuetudini degli stessi luoghi, nei quali «sono stati, et sono soliti tutti i servienti di giustizia di portare liberamente le $\operatorname{armi} \varkappa^{30}$.

In sostanza il ragionamento del Senato piemontese finiva per risolversi in una critica (neanche troppo velata) al modo in cui Vittorio Amedeo II aveva legiferato nel 1699. Se dopo la prima comunicazione del re era ormai indiscutibile che la volontà sovrana fosse nel senso di comprendere anche la categoria cui apparteneva Revello nell'applicazione della norma, i senatori sostennero che la redazione generica di quest'ultima non la rendesse per nulla chiara ed al contrario suscettibile di giustificabili fraintendimenti. Per tale ragione la magistratura torinese arrivò a suggerire al sovrano «che per gli stessi motivi sovra considerati sarebbe spediente il diffidare per altri casi simili detti servienti, ed ufficiali di giustizia, colla pubblicazione di qualche manifesto, $\mathrm{o}$ in altro modo, che le paresse più conveniente ${ }^{31}$.

Per come si è sviluppata la vicenda, anche al netto della possibile fondatezza giuridica delle argomentazioni senatorie ${ }^{32}$, non stupisce che le stesse siano state percepite da Vittorio Amedeo II come delle vere e proprie provocazioni. Per rispondere adeguatamente al Senato il re chiese alcuni pareri sulla disobbedienza della sua magistratura a importanti personaggi vicini alla corte che avevano recentemente preso parte ai lunghi lavori di riforma del sistema giuridico sabaudo come il savoiardo Pierre Mellarède, Giuseppe Spirito Riccardi, Ottavio Cotti, Bartolomeo Giusiana e Giovanni Cristoforo Zoppi ${ }^{33}$. Questi ultimi concordarono,

28 Il proemio dell'editto del 4 luglio 1699 recitava infatti: «Dopo aver fatto subire il dovuto castigo alli ribelli e malfattori della provincia di Mondovì, volendo accertare la continuazione della di lei quiete e tranquillità, abbiamo rivolto il pensiero a rinnovare, e far questi altri stabilimenti, che devono influire a questo fine», Duboin, 1830, p. 26.

${ }^{29}$ ASTo, Sez. Corte, Materie Giuridiche, Materie giuridiche per categorie, Senato di Piemonte, m. 2, n. 16, Lettera del Senato di Piemonte a S.M., cit.

30 Ibidem.

31 Ibidem.

32 Non prive di fondamento, come fa notare Genta, 1983, p. 21.

33 Viora, 1928, pp. 48-155. Per una biografia essenziale di questi importanti giuristi si veda Dionisotti, 1881, pp. 203-204, 289, 397, 482. Su Zoppi si veda anche Bonzo, 2013, 
a sostegno della posizione regia, nel ritenere infondata la tesi della mancanza di dolo del Revello e si impegnarono a contestarla punto per punto. In particolare essi sottolinearono la presenza nella vicenda di diversi elementi idonei, se considerati nel complesso, ad escludere la buona fede dell'arrestato. Alcuni di questi elementi riguardavano la particolare storia della provincia di Mondovì e dei moti che l'avevano interessata pochi decenni prima, ma altri riguardavano direttamente la figura del fiscale (ad esempio il coinvolgimento del padre nei recenti tumulti), i suoi precedenti penali e le circostanze del suo reato, consumato al di fuori dei luoghi di sua giurisdizione e in occasione di una festività religiosa (quando dovrebbe essere ancora più insolito portare armi) ${ }^{34}$.

Anche alla luce di questi pareri, e richiamandosi ancora una volta al testo dell'editto del 1699, il sovrano replicò aspramente al Senato, asserendo che la soluzione della vicenda giuridica di Revello fosse in realtà «facile, e chiara» ${ }^{35}$. Inoltre ricordò che proprio il citato editto proibiva espressamente a qualunque giudice di dispensare o moderare la pena prevista per la sua violazione ${ }^{36}$ : proprio per questa ragione - continuava il re rivolgendosi ai suoi senatori - «siamo certi che avrete un proporzionato pentimento d'esservi avanzati con la seconda rappresentanza a richiedere le nostre determinazioni in un affare, per cui tanto chiaramente resistevano gli editti» ${ }^{37}$.

Come ha notato Enrico Genta, in questa seconda risposta il sovrano non si è limitato ad accusare i senatori di insubordinazione ma si è spinto fino a rimproverar loro di non essere in grado di interpretare correttamente la legge ${ }^{38}$.

Alcuni fra gli stessi giuristi interpellati a suo favore da Vittorio Amedeo II hanno in effetti letto l'intera vicenda come una contrapposizione tra un'applicazione

pp. 2093-2094. Su Mellarède si veda Merlotti, 2009, pp. 316-319 e Merlin, 2016, pp. 157-170.

${ }^{34}$ Un riassunto dei pareri dati sul punto da Riccardi, Cotti e Giusiana è riportato a margine (con i nomi degli autori celati sotto gli pseudonimi di Sempronio, Tizio e Mevio) in ASTo, Sez. Corte, Materie Giuridiche, Materie giuridiche per categorie, Senato di Piemonte, m. 2, n. 33, 1, Relazione della causa criminale, e fatti concernenti la medesima, cit., ff. 7 v. $-10 \mathrm{v}$.

${ }^{35}$ ASTo, Sez. Corte, Materie Giuridiche, Materie giuridiche per categorie, Senato di Piemonte, m. 2, n. 33, 17, Copia di biglietto di S.M. al Senato di Piemonte; con cui gli rimprovera che dopo ricercata e avuta la regia intenzione siasi rivoltato con voti contrari ad assolvere il fiscale Revello, 30 aprile 1723.

36 «Per ultimo proibiamo a detti giudici di dispensarsi dall'esecuzione di quest'editto con la moderazione delle pene, de quali in esso, sotto pena d'esser decaduti ipso facto dal loro ufficio, et altra a Noi arbitraria, abolendo a tal effetto tutti gli editti antecedenti concernenti quanto sopra, e particolarmente quello delli 12 settembre scorso in quanto possano esser ripugnanti al presente»; Duboin, 1830, p. 34.

37 ASTo, Sez. Corte, Materie Giuridiche, Materie giuridiche per categorie, Senato di Piemonte, m. 2, n. 33, 17, Copia di biglietto di S.M. al Senato di Piemonte, cit.

38 Genta, 1983, p. 21. 
equitativa del diritto (operata dai senatori) ed una invece rigorosa (pretesa dal re $)^{39}$. A ben vedere la questione era però più profonda. II problema giuridico sotteso verteva infatti sull'estensione del potere interpretativo riconosciuto al Senato (ma il discorso potrebbe essere ampliato più in generale anche alle altre magistrature sabaude) sulla normativa regia.

Nel regno di Sardegna, all'inizio del XVIII secolo, il potere di interpretazione era ancora considerato a tutti gli effetti come una «manifestazione della funzione legislativa ${ }^{40} \mathrm{e}$, in quanto tale, riservato esclusivamente al sovrano. Proprio per questa ragione nel proemio della prima edizione delle Regie Costituzioni Vittorio Amedeo II si premurò di specificare quanto segue:

Non potrà verun Senato, Magistrato, o Tribunale, benché Supremo, usar sopra le medesime, per qualunque caso, che occorra, interpretazione alcuna, non volendo che sieno soggette a Limitazione, Dichiarazione, Ampliazione, o Moderazione, che non dipenda da Noi, o da' Nostri Successori ${ }^{41}$.

La pretesa del re di riuscire ad imporre la propria volontà legislativa su una magistratura non sempre concorde non era certo nuova negli Stati sabaudi, tanto che una disposizione generale, analoga a quella appena citata, si può riscontrare già nei Decreta seu Statuta di Amedeo VIII $(1430)^{42}$ oltre che nelle varie previsioni particolari presenti in diversi editti (compreso quello del 1699).

Quanto appena esposto fu da Vittorio Amedeo II efficacemente riassunto nella frase conclusiva che rivolse al Senato in risposta alla seconda rappresentanza: «Ricordatevi intanto, che nè Magistrati è riposta e la necessità, e la gloria di dare esecuzione alle Leggi, e non di variarle ${ }^{43}$.

Si può quindi convenire con quanto asserito (oltre cent'anni dopo) da un altro magistrato e attento studioso della storia sabauda come Carlo Dionisotti, secondo cui «il motivo dei rigori del Re verso taluni membri del Senato non fu per aver pronunciato una sentenza secondo la loro coscienza, ma per aver chiesta l'interpretazione sovrana della legge, e d'averla quindi disconosciuta» ${ }^{44}$.

Nel caso di specie infatti il Senato, facendo ricorso a principi generali, era arrivato ad individuare in via interpretativa la necessità di un elemento costitutivo ulteriore per la configurabilità della fattispecie dell'editto del 1699 imputata al Revello, ovvero il dolo. In questo modo era stata totalmente disattesa la lettura

\footnotetext{
${ }^{39}$ ASTo, Sez. Corte, Materie Giuridiche, Materie giuridiche per categorie, Senato di Piemonte, m. 2, n. 33, 1, Relazione della causa criminale, e fatti concernenti la medesima, cit., f. 7 v.

40 Soffietti, Montanari, 2008, p. 58; in generale Alvazzi del Frate, 2000, pp. 72 ss.

${ }^{41}$ RR.CC., 1723, § 2, proemio.

42 Soffietti, 2019, pp. 391-406.

${ }^{43}$ ASTo, Sez. Corte, Materie Giuridiche, Materie giuridiche per categorie, Senato di Piemonte, m. 2, n. 33, 17, Copia di biglietto di S.M. al Senato di Piemonte, cit.

${ }^{44}$ Dionisotti, 1881, p. 285.
} 
oggettivistica della norma pretesa invece dal re, che prescindeva da ogni questione relativa all'elemento soggettivo dell'autore della violazione. Se quindi in apparenza la questione poteva effettivamente apparire come una mera applicazione di una sorta di principio di equità (intesa come giustizia nel caso concreto) siamo in realtà dinnanzi ad un problema giuridico assai più ampio e antico. D'altronde il fatto che il problema trascendesse la banale vicenda giudiziaria del fiscale di Monastero era stato in parte colto anche dai giuristi interpellati a suo sostegno dal sovrano, secondo i quali «E qui è pur anche da avvertirsi, che questi termini d'assoluzione dal delitto del porto d'armi, de quali usa il Senato, portano l'esclusione dell'incorso nella pena dell'Editto, non solamente del Revello, ma di tutti gl'altri fiscali, perché con questo è decisa la causa per tutti» ${ }^{45}$.

II Senato avrebbe quindi dovuto dare esecuzione alla normativa regia, applicandola al fiscale monregalese con severità, ed eventualmente, nel caso in cui avesse ritenuto esagerata la pena di morte, avrebbe dovuto sospendere l'esecuzione della sentenza rimettendosi alla clemenza del sovrano, evitando così di interpretare la legge autonomamente in modo difforme dal re. In effetti che la vicenda sottendesse una questione di giustizia sostanziale era abbastanza evidente, e non solo al Senato: certamente Revello era stato incauto e superficiale e nel corso degli anni aveva violato diversi obblighi di natura burocratica e amministrativa, ma la morte pareva del tutto abnorme rispetto alla gravità e lesività della condotta posta in essere. Questo era però un problema di cui avrebbe dovuto occuparsi direttamente il re, in via di grazia. Nel sostenere questa tesi, Riccardi volle ricordare nel suo parere un caso simile occorso l'anno precedente ad un certo Antonio Gonella di Magliano il quale era stato condannato a morte in contumacia per aver esploso un colpo di pistola in aria "per puro modo d'allegrezza all'occasione che una sposa usciva da Messa ${ }^{46}$ e la cui pena era stata successivamente commutata in via di grazia dal sovrano in 5 anni di galera.

Sarebbe stato infatti istituzionalmente accettabile se il Senato avesse chiesto a Vittorio Amedeo II di cambiare il destino di Revello applicando l'equitas, ma altra cosa era pretendere di mettere in discussione, in via interpretativa, la portata di un editto.

\section{Le conseguenze del caso Revello nel contesto della riforma delle magi- strature sabaude}

La forte tensione istituzionale tra il re ed il Senato di Piemonte poteva quindi dirsi conclusa, quantomeno da un punto di vista strettamente giuridico. Era però fisiologico che questa vicenda finisse per avere conseguenze anche sul piano po-

${ }^{45}$ ASTo, Sez. Corte, Materie Giuridiche, Materie giuridiche per categorie, Senato di Piemonte, m. 2, n. 33, 1, Relazione della causa criminale, e fatti concernenti la medesima, cit., f. 10 r.

${ }^{46}$ Ivi, f. 12 v. 
litico. Vittorio Amedeo II non poteva infatti soprassedere di fronte alla presa di posizione di una magistratura così importante che aveva dimostrato di avere e di pretendere una così ampia autonomia.

Tuttavia decidere se e come punire il Senato per la sua insubordinazione era una questione delicata. Anche su questo aspetto il re chiese ausilio ai giuristi a lui fedeli che oscillarono tra posizioni più intransigenti (come la proposta della sospensione dallo stipendio per tutti i senatori oltre alla relegazione per il presidente Pietro Paolo Francesco Leone) ed altre meno severe, ma che comunque subordinavano la concessione del perdono regio ad atti umilianti, come il recarsi a piedi dal sovrano in processione senza alcun segno onorifico al fine di chiederne la grazia ${ }^{47}$.

Particolarmente interessante è il parere che redasse Nicolò Pensabene, già reggente del Supremo Consiglio di Sicilia e, in quel momento, Conservatore dell'Università degli studi di Torino ${ }^{48}$. II giurista palermitano non si limitò a commentare le posizioni espresse dagli altri giuristi circa le misure da assumere nei confronti dei senatori, ma raccolse e inviò al sovrano il resoconto di una serie di provvedimenti presi nel corso dei secoli dai re di Francia contro il Parlamento di Parigi. Questo non stupisce: a prescindere dai rapporti diplomatici piuttosto oscillanti ${ }^{49}$, la politica assolutistica francese ha rappresentato un modello di riferimento importante per l'opera accentratrice di Vittorio Amedeo I $^{50}$ e gli stessi Senati sabaudi erano stati costituiti sulla scorta delle Cours de Parlement introdotte durante l'occupazione francese del XVI secolo ${ }^{51}$.

È interessante riportare alcuni di questi casi appositamente selezionati per Vittorio Amedeo II dal Pensabene, il quale ricordava quanto segue:

[...] Nell'anno 1525 li 25 di luglio. II Re essendo andato alla Corte a tenere trono di giustizia per pronunciare l'arresto per difetto dato contro il sig. Carlo de Bourbon accusato di peccato di fellonia, rebellione e di lesa maestà, dopo la detta pronunciazione il sig. Giovanni Roberto segretario di Stato diede al Conservatore della Corte un editto delli 24 del detto mese di luglio del 1525 proibendo al Parlamento di non mischiarsi tra l'affari di Stato, ne in altra cosa che della Giustizia, obbligando alla detta Corte di prendere tutti gl'anni le lettere del loro potere, e delegazione. Proibisce di riconoscere le materie arcivescovili, e abbazie, cassazione de limitazioni, e restrizioni apportati per la detta Corte alla Regenza della Regina Madre, et confermando tutto quello che la detta Regina, costituendola regente in tempo

\footnotetext{
47 Genta, 1986, p. 393.

48 Sull'esperienza di Pensabene come Conservatore dell'Università e sugli scontri che per questa ragione ebbe con il Senato negli anni venti del XVIII secolo si rimanda a Traverso, 2020, pp. 191-210.

${ }^{49}$ Emblematica è, ad esempio, proprio l'esperienza della Guerra del sale ed il ruolo in essa indirettamente ricoperto da Luigi XIV; si veda Lombardi, 1986, pp. 116-118.

50 Stumpo, 2002, p. 247.

51 Soffietti, Montanari, 2008, pp. 44-45.
} 
di sua absenza con medesima autorità, ordinando che tutto quello ch'era stato fatto nella detta corte contro gl'ordini della regente, li fossero portati per essere cancellati.

Proibisce alla detta Corte di modificare gl'editti, e che procederanno per avvertimento al Re.

Declara ancora ch'il Parlamento non ha alcuna Giurisdizione sopra il cancelliere, e ch'il registro di quello che ha stato fatto contra di lui al Parlamento, sia portato al Re, e sia cancellato e cassato. [...]

Nell'anno 1564 delli 23 di gennaio, il Re ordinò che l'ordinazioni da lui fatte, e rinnovate fossero trattenute, et ancora in tutte le altre cose che comanderà vuole che fosse obbedito, e che la Corte solamente amministrasse Giustizia a tutti i suoi soggetti nel farli vivere in pace gl'uni con gl'altri in riposo e tranquillità e sicurezza delle loro vite, e beni, e non vuole che la corte si mischiasse di qua innanzi in altri affari, che in quelli nelli quali lui comanderà, e qui dipenderà del suo stato nella distribuzione della Giustizia, e fare trattenere, e obbedire gl'editti del Re, non volendo però che in questo la Corte risparmiasse uno solo, di quelli che contravvenissero e che ne facesse un buono esempio, acciò che il Re fosse meglio obbedito nell'avvenire che non ha in passato. Ordina ancora al Procuratore generale di fare il suo dovere, acciò ch'il tutto fosse inviolabilmente osservato, e che nessuna delle sue Corti Parlamentari potesse dare nell'avvenire arresto contro l'ordinazioni, et editti senza averne ricevuto ordine, o comando.

Nell'anno 1591 al 18 dicembre, il Re essendo mal soddisfatto del procedimento del Parlamento nel dare delle difficoltà, e nel rifiutare di verificare molti editti di sua propria mano scritti, e che aventia inteso le sue raggioni, tutte le volte che le trovava buone come sempre farebbe quando vedeva che la Corte gli porteria l'obbedienza che gli deve, ma vedendo che la Corte se ne aveva abusato dopo la sua venuta alla Corona, e che non lascia di continuare indisprezzare gli suoi comandi e che l'aveva voluto dare questo onore non usitato di scriverci di sua propria mano, e comandarli d'obbedire di qua innanzi alli suoi ordini, o altrimenti li farà conoscere che ha avuto mai Re che s'avesse fatto meglio obbidire, che lui stesso ${ }^{52}$.

Come si può notare, gli esempi portati all'attenzione di Vittorio Amedeo II attenevano a casi (quasi tutti temporalmente collocati nel XVI secolo) in cui i sovrani francesi si erano imposti sul Parlamento parigino obbligando i suoi membri a recepirne gli editti o a non ingerirsi in questioni politiche che esulavano dalle competenze più propriamente giudiziarie. Anche le supreme magistrature sabaude, secondo un modello classico delle grandi corti sorte in età moderna ${ }^{53}$, presentavano una commistione evidente tra competenze giuridiche e ammini-

\footnotetext{
${ }^{52}$ ASTo, Sez. Corte, Materie Giuridiche, Materie giuridiche per categorie, Senato di Piemonte, m. 2, n. 20, Parere del Marchese Regente Pensabene sovra la condatta del Senato nella Causa dell'avvocato Revello fiscale del Mondovì che non ha voluto condannare à morte secondo il disposto dall'editto contro il porto d'armi prohibite. Con alcune memorie toccanti la maniera con cui li Re di Francia hanno castigato il Parlamento, tirate dalli Registri del medesimo.
}

53 Sbriccoli, Bettoni, 1993; Ascheri,1989; Gorla, 1969, pp. 3-39. 
strative e nel corso del XVII secolo in diverse occasioni erano arrivate anche a

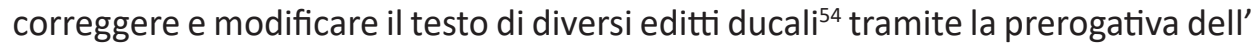
"interinazione" 55 . Ridimensionare in generale le attribuzioni latu sensu politiche di queste istituzioni, riaffermando le prerogative regie, rientrava certamente tra le aspettative di un sovrano con tendenze assolutistiche come Vittorio Amedeo II: questo obiettivo era stato in parte raggiunto proprio con l'emanazione di una nuova normativa che lasciava meno arbitrio ai Senati, ma "I'affare Revello" offriva ora anche l'occasione di disfarsi di alcuni senatori percepiti come non allineati all'idea che il re aveva del ruolo della magistratura nel regno di Sardegna.

Nel mese di settembre 1723 Vittorio Amedeo II comunicava quindi al Senato, tramite il Mellarède, le proprie determinazioni. Veniva disposta l'immediata sospensione dalle funzioni del presidente Pietro Paolo Francesco Leone, dell'avvocato fiscale generale Gerolamo Vitale Pasta e del senatore che aveva fatto da relatore nella causa di Revello Ignazio Dionigi Meynier ${ }^{56}$. Inoltre al presidente Leone, "che era più degli altri obbligato ad invigilare per l'osservanza dei suoi ordini ${ }^{57}$, venne ordinato di trasferirsi entro 24 ore o a Livorno o a Leyni, nell'attesa di ulteriori decisioni sulla sua sorte.

Le conseguenze disciplinari contro il Senato non si limitarono a colpire i giudici che avevano preso parte alla deliberazione su Revello. Ad essere oggetto dei provvedimenti sanzionatori del re fu anche Maurizio Ignazio Graneri, importante giurista sabaudo che nel 1713 era stato «promosso al grado di Presidente nel Senato di Piemonte in considerazione del zelo per il Real servizio ${ }^{58}$. Pur non avendo fatto parte del collegio che assolse il fiscale di Monastero, Graneri aveva comunque affermato pubblicamente che Vittorio Amedeo II aveva utilizzato questa causa come pretesto per rimuovere dal Senato alcuni elementi sgraditi ${ }^{59}$. Sempre a lui vennero inoltre attribuite diverse altre prese di posizione sulla sen-

${ }^{54}$ Per un esempio relativo ad alcuni editti in materia di giurisdizione universitaria si veda Traverso, 2020, pp. 179-191.

${ }^{5} \mathrm{Su}$ questa particolare funzione senatoria e in generale sulle attribuzioni "costituzionali" delle supreme magistrature sabaude si veda Pene Vidari, 2016, pp. 75-83; Soffietti, Montanari, 2008, pp. 29-51, 75-95; Merlin, 1982, pp. 35-94; Lombardi, 1962, pp. 1-40; Viora, 1928, pp. 151-155; Lattes, 1908, pp. 1-47.

${ }^{56}$ ASTo, Sez. Corte, Materie Giuridiche, Materie giuridiche per categorie, Senato di Piemonte, m. 2, n. 33, Copia di lettera, 17 settembre 1723.

57 Ibidem.

58 ASTo, Sez. Corte, Materie Giuridiche, Materie giuridiche per categorie, Senato di Piemonte, m. 1 Prima d'addizione, n. 3, Stato degli uffiziali del Senato di Piemonte, colla data delle loro costituzioni, finanze pagate, e loro stipendi, f. 5.

${ }^{59}$ ASTo, Sez. Corte, Materie Giuridiche, Materie giuridiche per categorie, Senato di Piemonte, m. 1 d'addizione, n. 4, Relazione del conte Mellarede, copia di lettere del medesimo al presidente marchese Graneri, ed un ricavo de' discorsi tenuti da questo riguardanti il suo rifiuto di andare a riempire la presidenza del Senato di Nizza, a cui era stato da S.M. destinato per allontanarlo dal Senato di Piemonte per diverse calunnie al medesimo 
tenza di Revello verso le quali il sovrano non poteva restare indifferente: Graneri venne descritto come un uomo potente e "prepotente», capace di condizionare non solo la seconda classe del Senato (che presiedeva) ma l'intera corte; in sostanza venne dipinto come il vero istigatore dell'insubordinazione della magistratura senatoria ${ }^{60}$. All'epoca dei fatti (1723) Graneri aveva ormai 59 anni ed aveva ricoperto importanti ruoli diplomatici e giudiziari ${ }^{61}$.

Dopo questi fatti, per allontanarlo dal Senato torinese, nell'ottobre 1723 Vittorio Amedeo II lo nominò presidente del Senato di Nizza. Si trattava di un trasferimento chiaramente punitivo che avrebbe costretto Graneri ad abbandonare la capitale del regno ed assumere una carica assai meno prestigiosa di quella che già ricopriva. Graneri rifiutò, adducendo come ragione la necessità di assistere la moglie, Elena Margherita Valperga di Rivara, che versava in precarie condizioni di salute $^{62}$. A fronte dell'assoluta chiusura verso qualunque nomina che comportasse uno spostamento da Torino (anche se la destinazione non fosse stata il Senato di Nizza, ma i più vicini omologhi di Pinerolo o Casale, come suggeritogli dal Mellarède) Graneri venne infine relegato a Cherasco il 25 ottobre $1723^{63}$.

II rifiuto di obbedire ad un ordine diretto di Vittorio Amedeo II costò caro all'ex senatore. In ragione di questo comportamento nel 1725 l'avvocato fiscale Berterini $^{64}$ suggeriva al re di revocare la sua relegazione, ma di continuare comunque a non concedergli i benefici economici connessi alla sua posizione e di imporgli una vita strettamente privata ${ }^{65}$. Quanto invece alla posizione di Pietro Paolo Francesco Leone nel medesimo parere l'avvocato fiscale suggerì una maggior clemenza, «essendo certissimo che la sospensione, e relegazione d'un presidente reggente il Senato è una pena gravissima ancorché fosse praticata per minor tempo di quello che l'abbia patita il presidente Leone» ${ }^{66}$.

fatte, e particolarmente di essere stato il motore principale che il Senato non ha voluto condannare a morte l'avvocato Revello fiscale del Mondovì. Dalla quale relazione risulta che il detto presidente Graneri per la sua inubbidienza agli ordini sovrani venne esiliato da Torino, e relegato a Cherasco.

${ }^{60}$ ASTo, Sez. Corte, Materie Giuridiche, Materie giuridiche per categorie, Senato di Piemonte, m. 1 Prima d'addizione, n. 4, Ricavo dei discorsi tenuti dal presidente Graneri sovra il fatto del fiscale Revello.

61 Merlotti, 2002, pp. 535-536; Dionisotti, 1881, pp. 284-285.

62 ASTo, Sez. Corte, Materie Giuridiche, Materie giuridiche per categorie, Senato di Piemonte, m. 1 d'addizione, n. 4, Relazione del conte Mellarede, cit.

63 Ibidem.

${ }^{64}$ Su Giovanni Battista Berterini si veda Viora, 1928, p. 75.

65 ASTo, Sez. Corte, Materie Giuridiche, Materie giuridiche per categorie, Senato di Piemonte, m. 2, n. 33, 8 aprile 1725, Breve compendio del fatto, e dei sentimenti dati a S.M. nella causa del fiscale Revello, col parere sopra la relegazione, e sospensione del presidente Leone, Marchese Graneri, e cav. Pasta.

66 Ibidem. 
I membri più in vista del Senato di Torino che nel 1723 cercarono di opporsi al re vennero quindi allontanati dall'amministrazione dello Stato. Alcuni, come il Graneri, non vi rientreranno più (anche per ragioni di età) e termineranno la propria esistenza lontano dalla vita pubblica, altri invece vennero successivamente riabilitati da Vittorio Amedeo $\|^{67}$. Infine, nel novembre 1723 , il re provvide a nominare 7 nuovi membri del Senato da lui appositamente selezionati, modificando così sensibilmente la fisionomia dell'istituzione ${ }^{68}$.

Come lo era stata anni prima la Guerra del Sale, anche la breve "fronda" senatoria era stata repressa con risolutezza ed era ormai chiaro che il Senato di Torino non avrebbe più potuto contare su quella autonomia giuridica e politica di cui, ancora nel XVII secolo, aveva ampiamente goduto.

\section{Conclusioni. Vittorio Amedeo Il e il caso Revello: despota o innovatore?} La lettura della storiografia sabauda ottocentesca

Il giudizio dato dalla storiografia sabauda ottocentesca sulla condotta tenuta da Vittorio Amedeo II in questa vicenda non è stato certo positivo.

Presentando il caso nella sua Storia della legislazione italiana Federigo Sclopis non mancò di notare che «Talvolta, ed era tristissimo esempio, l'autorità del principe credendo di interporsi a pro del pubblico bene, piombava con tutto il suo peso sull'officio del giudice ${ }^{69}$.

Domenico Carutti si pose sulla stessa linea critica e nella Storia del regno di Vittorio Amedeo II affermò:

II peggio fu che Vittorio Amedeo II non rispettò sempre la libertà dei tribunali, e ne preoccupò talune volte il giudizio con ammonizioni, regi biglietti ed altri argomenti simiglianti [...]. La patria magistratura per intierezza e dignità di costume [...] seppe resistere ai principi quando per errore o debolezza di consiglio si discostavano dal retto sentiero nelle cose di giustizia ${ }^{70}$.

La vicenda di Revello si prestava bene a queste letture e anche un importante storico come Luigi Cibrario vide in questa situazione un esempio delle tendenze assolutistiche del primo re di Sardegna; come egli osservò:

Vittorio Amedeo, principe grande, ma principe di voglie assolute, s'altri mai fu, dimenticava che l'indipendenza de' magistrati è la guarentigia del trono: che la delegazione ai medesimi fatta d'amministrare la giustizia in nome e luogo del sovrano, non può ammettere restrizioni circa alla pienissima libertà del voto $[\ldots]^{71}$.

\footnotetext{
67 È il caso di Ignazio Dionigi Meynier, vedi Cerutti, 2003, p. 186.

68 Genta, 1983, p. 27.

69 Sclopis, 1863, p. 441.

70 Carutti, 1856, pp. 395-396.

${ }^{71}$ Cibrario, 1846, p. 726. Una succinta descrizione della vicenda è anche presente in Cibrario, 1855, p. 454.
} 
Si tratta di valutazioni che non possono dirsi del tutto errate, ma forse parziali ed eccessivamente semplicistiche. Nell'esaminarle bisogna tenere presente che l'intento di questi autori, quasi tutti giuristi per formazione o professione (Sclopis e Cibrario in particolare) era di esaltare la magistratura sabauda contrapponendola alle ingiuste ed arbitrarie pretese del re. Siamo di fronte a giuristi e uomini di Stato che giudicano, secondo le categorie del loro tempo, i loro predecessori, in positivo (come nel caso di Graneri e Leone), ma anche in negativo. Impietosa è, ad esempio, la critica che Cibrario riservò a Pensabene, Mellarède, Riccardi e agli altri personaggi che sostennero le ragioni del re, che vennero descritti come "ministri cortigiani» ${ }^{72}$ capaci di adulare ma non di consigliare correttamente il sovrano.

Chi invece venne agiograficamente esaltato fu Graneri che finì per assurgere all'emblema di una magistratura libera che era stata spezzata, ma che non si era mai piegata alle pretese del re. Si arrivò anche ad esasperare le presunte ritorsioni che dovette subire, sostenendo ad esempio che durante il periodo della relegazione a Cherasco gli fosse stato proibito di recarsi al capezzale della moglie ${ }^{73}$, circostanza smentita da più recenti studi ${ }^{74}$.

L'azione di Vittorio Amedeo II poteva rappresentare, agli occhi di un osservatore del XIX secolo, un'ingerenza intollerabile, ma rientrava in un programma preciso di riforma del sistema magistratuale portato indefessamente avanti dal sovrano. Una magistratura che, all'inizio del XVIII secolo, presentava alcune caratteristiche ormai anacronistiche, a cominciare dalle modalità di selezione dei suoi membri. Fino al regno di Vittorio Amedeo II infatti la carica di senatore rientrava tra quelle alienabili dal fisco e trasmissibili ${ }^{75}$. Gran parte dei senatori, compresi i protagonisti dell'assoluzione di Revello, avevano tutti ottenuto la loro nomina in questo modo: il presidente Leone era subentrato nella carica a suo padre Guglielmo ${ }^{76}$, Granieri al fratello Carlo Emanuele, Meynier al senatore Pietro Erasmo Hondio ${ }^{77}$, l'avvocato fiscale Girolamo Vitale Pasta al padre ${ }^{78}$.

La loro sostituzione con personaggi scelti direttamente dal re rientrava quindi in un programma più ampio volto a rendere più efficiente e centralizzato il siste-

72 « [...] crediamo doversi ascrivere a quei ministri cortigiani, che per rendersi necessari al principe usano d'adularne le passioni, e invece di temperare con rispettosi consigli le ire tanto pericolose di chi può ciò che vuole [...] usano all'incontro inasprirne la fierezza, armarne di più velenose punte gli sdegni», Cibrario, 1846, p. 727.

73 Carutti, 1856, pp. 395-396.

${ }^{74}$ La moglie Elena Maria Valperga di Rivara morirà infatti solo nel 1728 e non durante gli anni passati al confino dal marito; Merlotti, 2002, p. 536.

75 Genta, 1983, p. 67.

76 Dionisotti, 1881, p. 337.

77 Ivi, p. 334.

78 ASTo, Sez. Corte, Materie Giuridiche, Materie giuridiche per categorie, Senato di Piemonte, m. 1 Prima d'addizione, n. 3, Stato degli uffiziali del Senato di Piemonte, cit., ff. 4-5, 12, 20. 
ma burocratico-giudiziario sabaudo: come è stato notato non si tratta semplicemente dei capricci di un sovrano con tendenze tiranniche ed arbitrarie, ma siamo piuttosto di fronte ad uno dei problemi cruciali della formazione dello stato moderno ${ }^{79}$.

D'altronde il Senato di Torino non fu l'unica istituzione giudiziaria che in quegli anni venne direttamente colpita dal re: tra il 1719 ed il 1724 Vittorio Amedeo II sostituì infatti anche gran parte dei membri della Camera dei conti. Con un provvedimento del 28 dicembre 1719 vennero licenziati tutti gli ufficiali della Camera per alcune precedenti mancanze ai loro doveri e il 7 gennaio dell'anno successivo venne emanato un nuovo regolamento per questa magistratura contestualmente alla nomina dei nuovi membri ${ }^{80}$.

Ecco che in pochi anni il sovrano riuscì a riaffermare il proprio controllo sull'alta magistratura sabauda con una serie di incisivi interventi normativi e politici che si collocavano in una più ampia serie di riforme dell'apparato burocratico regno soprattutto di ordine fiscale e istituzionale.

È quasi superfluo specificare che siamo ancora molto lontani dalla creazione di un ordinamento giudiziario organizzato e accentrato secondo i canoni giuridici contemporanei. Probabilmente a un tal risultato nel regno di Sardegna si giungerà infatti solo un secolo dopo, con le riforme di Carlo Alberto del 1847-1848. Tuttavia la razionalizzazione del sistema tentata nella prima parte del XVIII secolo ha rappresentato un momento fondamentale per la storia giuridica degli Stati sabaudi, e segnerà l'organizzazione della magistratura per tutto il secolo e buona parte di quello successivo.

\section{Bibliografia}

Aimerito F., 2018: Ricerche sul "Consiglio di Stato e dei Memoriali" degli Stati sabaudi. Percorsi fra equità, diritto e politica (secoli XVI-XIX), Torino, Giappichelli

Alvazzi del Frate P., 2000: L'interpretazione autentica nel XVIII secolo. Divieto di interpretatio e "Riferimento al legislatore» nell'illuminismo giuridico, Torino, Giappichelli

Archivio di Stato di Torino (ASTo): Sezione Corte, Materie Giuridiche, Materie giuridiche per categorie, Camera dei Conti di Piemonte, m. 1

Archivio di Stato di Torino (ASTo): Sezione Corte, Materie Giuridiche, Materie giuridiche per categorie, Senato di Piemonte, mazzo 1 Prima addizione

Archivio di Stato di Torino (ASTo): Sezione Corte, Materie Giuridiche, Materie giuridiche per categorie, Senato di Piemonte, mazzo 2 (inventariato)

79 Genta, 1986, p. 391.

${ }^{80}$ ASTo, Sez. Corte, Materie Giuridiche, Materie giuridiche per categorie, Camera dei Conti di Piemonte, m. 1, f. 38. 
Ascheri M., 1989: Tribunali, giuristi ed istituzioni: dal medioevo all'età moderna, Bologna, II Mulino

Bianchi P., Merlotti A., 2017: Storia degli Stati sabaudi (1416-1848), Brescia, Morcelliana

Bonzo C., 2013: Zoppi, Giovanni Cristoforo, in Dizionario biografico dei giuristi italiani (XII-XX secolo), II, Bologna, II Mulino

Briegel F., Milbach S. (ed.), 2016: Les Sénats des Etats de Savoie. Circulations des pratiques judiciaires, des magistrats, des normes (XVI-XIX siècles), Roma, Carocci

Carutti D., 1856: Storia del regno di Vittorio Amedeo II, Torino, Paravia

Casana P., 1995: Un esempio di corte suprema nell'età del diritto comune. II Senato di Piemonte nei primi decenni di attività, Torino, Giappichelli

Cerutti S., 2003: Giustizia Sommaria. Pratiche e ideali di giustizia in una società di ancien Régime (Torino XVIII secolo), Milano, Feltrinelli

Cibrario L., 1855: Origini e progresso delle instituzioni della monarchia di Savoia, parte seconda, Torino, Dalla Stamperia Reale

Cibrario L., 1846: Storia di Torino, II, Torino, Alessandro Fontana

Dionisotti C., 1881: Storia della Magistratura piemontese, II, Torino, Roux e Favale

Duboin F.A., 1830: Raccolta per ordine di materie delle leggi, editti, manifesti, ecc. pubblicati dal principio dell'anno 1681 sino agli 8 dicembre 1798 sotto il felicissimo dominio della Real Casa di Savoia, tomo VI, volume VIII, Torino, Vittorio Picco

Genta E., 1986: Condanne, grazie e porto d'armi: proiezioni della Guerra del Sale sul riordinamento del Senato, in G. Lombardi (ed.), La Guerra del Sale (16801699). Rivolte e frontiere del Piemonte barocco, I, Milano, Franco Angeli

Genta E., 1983: Senato e senatori di Piemonte nel secolo XVIII, Torino, Deputazione Subalpina di storia patria

Gorla G., 1969: I "grandi Tribunali" italiani fra i secoli XVI e XIX: un capitolo incompiuto della storia politico-giuridica d'Italia, in "Quaderni del Foro Italiano", 1969

Lange A., 1986: La «seconda guerra del sale» (1698-1704). Esiliati e ribelli, in G. Lombardi (ed.), La Guerra del Sale (1680-1699). Rivolte e frontiere del Piemonte barocco, II, Milano, Franco Angeli

Lattes A., 1908: L'interinazione degli editti: studio di storia del diritto pubblico piemontese, Torino, Clausen

Lombardi G., 2003: I Ferrero nelle Guerre del sale (1680-1699): I'obbligo politico dalla "patria» cittadina allo Stato, in A. Merlotti (ed.), Nobiltà e Stato in Piemonte. I Ferrero d'Ormea, atti del convegno Torino-Mondovì 3-5 ottobre 2001, Torino, Silvio Zamorano editore 
Lombardi G., 1986: La Guerra del sale: Caleidoscopio di una Historia, in G. Lombardi (ed.), La Guerra del Sale (1680-1699). Rivolte e frontiere del Piemonte barocco, I, Milano, Franco Angeli

Lombardi G., 1962: Note sul controllo degli atti del sovrano negli Stati sabaudi ad opera delle supreme magistrature nel periodo dell'assolutismo, in "Annali della scuola speciale per archivisti e bibliotecari dell'Università di Roma", II, 1

Merlin P., 1982: Giustizia, amministrazione e politica nel Piemonte di Emanuele Filiberto. La riorganizzazione del Senato di Torino, in "Bollettino storicobibliografico subalpino", LXXX

Merlin P., 2016: Un savoiardo alla pace di Utrecht. Pierre Mellarède, tra diritto, diplomazia e politica, in F. leva (ed.), I trattati di Utrecht. Una pace di dimensione europea, Roma, Viella

Merlotti A., 2002: Graneri, Maurizio Ignazio, in Dizionario biografico degli italiani, 58, Roma, Treccani

Merlotti A., 2009: Mellarède de Betton, Pietro, in Dizionario biografico degli italiani, 73, Roma, Treccani

Micolo F., 1984: Le regie costituzioni: il cauto riformismo di una piccola Corte, Milano, Giuffrè

Mola di Nomaglio G., Melano G. (ed.), 2014: Utrecht 1713. I trattati che aprirono le porte d'Italia ai Savoia, Studi per il terzo centenario, Torino, Centro Studi Piemontesi

Mongiano E., 1991: Il Senato di Piemonte nell'ultimo trentennio dell'Antico Regime (1770-1798), in Dal Trono all'albero della libertà. Trasformazioni e continuità istituzionali nei territori del Regno di Sardegna dall'antico regime all'età rivoluzionaria, I, Roma, Ministero per i beni culturali e ambientali

Pene Vidari G.S., 2016: I Senati sabaudi: modelli e tendenze nel corso dei secoli, in F. Briegel, S. Milbach (ed.), Les Sénats des Etats de Savoie. Circulations des pratiques judiciaires, des magistrats, des normes (XVI-XIX siècles), Roma, Carocci

Pene Vidari G.S., 1986: La gabella del sale e le antiche franchigie monregalesi: un caso di esercizio del diritto di resistenza?, in G. Lombardi (ed.), La Guerra del Sale (1680-1699). Rivolte e frontiere del Piemonte barocco, I, Milano, Franco Angeli

Pene Vidari G.S. (ed.), 2001: Les Sénats de la Maison de Savoie. Ancien régime Restauration, Torino, Giappichelli

Ricuperati G., 2002: Introduzione, in G. Ricuperati (ed.), Storia di Torino. IV. La città fra crisi e ripresa (1630-1730), Torino, Einaudi

Ricuperati G., 2016: Una riflessione a premessa sul significato di Utrecht 1713. Italia, Europa, Mondo, in F. leva (ed.), I trattati di Utrecht. Una pace di dimensione europea, Roma, Viella 
RR.CC., 1723: Leggi e Costituzioni di S.M. da osservarsi nelle materie civili e criminali ne' Stati della M. S., tanto di quà che di là da monti e colli, Torino, Giovanni Battista Valetta

Sbriccoli M., Bettoni A. (ed.), 1993: Grandi tribunali e rote nell'Italia di Antico Regime, Milano, Giuffrè

Sclopis F., 1863: Storia della legislazione italiana, II, parte 2, Torino, Unione tipografico editrice

Soffietti I., 2019: "Reservata nobis facultate statuta declarandi»: I'interpretazione autentica degli Statuta Sabaudie, in M. Caesar, F. Morenzoni (ed.), La loi du prince. La raccolta normativa sabauda di Amedeo VIII (1430), I. Gli statuti sabaudi di Amedeo VIII del 1430. Un'opera legislativa di rilievo, Torino, Deputazione Subalpina di storia patria

Soffietti I., Montanari C., 2008: II diritto negli Stati sabaudi (secoli XV-XIX), Torino, Giappichelli

Stumpo E., 2002: Economia urbana e gruppi sociali, in G. Ricuperati (ed.), Storia di Torino. IV. La città fra crisi e ripresa (1630-1730), Torino, Einaudi

Symcox G., 1986': Vittorio Amedeo II, l'assolutismo sabaudo 1675-1730, trad. S. Patriarca, Torino, SEI

Traverso M., 2020: Il privilegium fori dell'Università di Torino tra i secoli XVII-XVIII. Ascesa e declino di una Magistratura, in "Rivista di Storia del Diritto Italiano", $\mathrm{XCIII,} 2$

Viora M.E., 1928: Le costituzioni piemontesi. Leggi e Costituzioni di S.M. il Re di Sardegna (1723-1729-1770). Storia esterna della compilazione, Torino, F.lli Bocca (rist. an. 1986, Savigliano, L’Artistica) 